\title{
An algorithm to compute the transfer function of a mechanical system
}

\author{
L Ribichini, V Leonhardt, H Lück and K Danzmann \\ Max Planck Institute for Gravitational Physics (Albert Einstein Institute) and University of \\ Hannover, Callinstrasse 38, D-30167 Hannover, Germany \\ E-mail: rxl@aei.mpg.de
}

Received 29 August 2003

Published 13 February 2004

Online at stacks.iop.org/CQG/21/S1247 (DOI: 10.1088/0264-9381/21/5/128)

\begin{abstract}
Reliable and efficient algorithms are needed to model complex mechanical systems such as multiple stage pendulum suspensions. A possible approach is the one based on the input-output formalism. This method allows us to model the system in a modular way. The basic objects (which are the individual stages) are modelled via matrices and then simply joined together through matrix multiplication. It is currently not widely used because it is known to be unstable. Some interesting results towards the stabilization of such algorithm are reported.
\end{abstract}

PACS number: $04.80 . \mathrm{Nn}$

\section{Introduction}

Gravitational wave (GW) detection is one of the major challenges of contemporary physics. For several decades attempts have been made using resonant bar detectors, working in a small frequency band around $1 \mathrm{kHz}$. In the last decade a complementary approach has become available: a kilometre-scale interferometer that will enable us to extend the detection band down to approximatively $10 \mathrm{~Hz}$ [1-4]. The expected limitation in this low-frequency region was considered the suspension thermal noise of the last pendulum stage [5]. This was the motivation that led us to start an independent smaller scale experiment to address this particular issue [6]: namely the direct measurement of the off resonant thermal noise by using an experimental setup as close as possible to that of the GW detectors. However, our experiment, and the various GW detectors as well [7], are reporting excess noise in this low-frequency region (up to $100 \mathrm{~Hz}$ ), a few orders of magnitude above the expected level. It is currently believed that one of the biggest noise sources in this frequency region is the seismic noise. Therefore new theoretical models and more realistic simulations are needed. These simulations should take all the six degrees of freedom of a rigid body and the couplings between them into account. 


\section{The theory}

The underlying theory is both simple and well known [8]. Each mechanical element in a mechanical chain can be represented by a matrix connecting input displacement and input force with the output displacement and output force in the frequency domain. We can write an input-output relation (in the frequency domain)

$$
\left(\begin{array}{c}
\tilde{x}_{\text {out }} \\
\tilde{F}_{\text {out }}
\end{array}\right)=A(\omega)\left(\begin{array}{c}
\tilde{x}_{\text {in }} \\
\tilde{F}_{\text {in }}
\end{array}\right)
$$

where $\tilde{x}_{\text {in }}$ is the input displacement, $\tilde{F}_{\text {out }}$ is the output force, and so on. For a system composed of a spring with elastic constant $k$ and a mass $m$, it is easy to write the following system of equations:

$$
\left(\begin{array}{c}
\tilde{x}_{\text {out }} \\
\tilde{F}_{\text {out }}
\end{array}\right)=\left(\begin{array}{cc}
1 & -\frac{1}{k} \\
m \omega^{2} & 1-\frac{m \omega^{2}}{k}
\end{array}\right)\left(\begin{array}{c}
\tilde{x}_{\text {in }} \\
\tilde{F}_{\text {in }}
\end{array}\right) .
$$

This is already one of the building blocks of a simple pendulum chain, so let us compute the transfer function (TF) of the system, which is defined as $\left|\tilde{x}_{\text {out }} / \tilde{x}_{\text {in }}\right|$ with the condition $\tilde{F}_{\text {out }}=0$. It is not difficult to obtain the following expression:

$$
\mathrm{TF} \equiv\left|\frac{\tilde{x}_{\mathrm{out}}}{\tilde{x}_{\mathrm{in}}}\right|=\left|\left(1-\frac{m \omega^{2}}{k}\right)^{-1}\right| .
$$

In general, given the matrix $A(\omega)$

$$
A(\omega)=\left(\begin{array}{ll}
a_{11} & a_{12} \\
a_{21} & a_{22}
\end{array}\right)
$$

one has

$$
\mathrm{TF}=\left|\frac{\operatorname{det} A(\omega)}{a_{22}}\right| .
$$

A big advantage of this method is that it is modular. That is, it offers the possibility of 'adding' various stages together, by just multiplying the respective matrices in the correct order. Unfortunately, it is also known that the algorithm is unstable [8], especially when higher order systems are simulated, such as when two degrees of freedom are coupled together and the matrix $A$ becomes a $4 \times 4$ matrix.

\subsection{A quick look at the problem with coupled degrees of freedom}

It is already known that the couplings between different degrees of freedom in a real pendulum (longitudinal motion and tilt motion) must be explicitly taken into account. So, let us consider a system in which two degrees of freedom are coupled together. In reality these degrees of freedom are one displacement and one angle (longitudinal motion and tilt motion, for example), but to keep the formulae as readable as possible, we will speak about two displacements coupled together. In this case, as we already said, the relevant matrices are of order 4 and we have

$$
\left(\begin{array}{c}
\tilde{X}_{\text {out }} \\
\tilde{G}_{\text {out }}
\end{array}\right)=\left(\begin{array}{cc} 
& -K^{-1} \\
\omega^{2} M & -\omega^{2} M K^{-1}
\end{array}\right)\left(\begin{array}{l}
\tilde{X}_{\text {in }} \\
\tilde{G}_{\text {in }}
\end{array}\right)
$$


where,

$$
\begin{aligned}
X_{\text {in }} \equiv\left(\begin{array}{l}
\tilde{x}_{1, \text { in }} \\
\tilde{x}_{2, \text { in }}
\end{array}\right) & G_{\text {in }} \equiv\left(\begin{array}{l}
\tilde{F}_{1, \text { in }} \\
\tilde{F}_{2, \text { in }}
\end{array}\right) \\
K \equiv\left(\begin{array}{ll}
k_{11} & k_{12} \\
k_{21} & k_{22}
\end{array}\right) & M \equiv\left(\begin{array}{cc}
m_{11} & 0 \\
0 & m_{22}
\end{array}\right) \equiv\left(\begin{array}{ll}
1 & 0 \\
0 & 1
\end{array}\right) .
\end{aligned}
$$

\section{Algorithm stabilization: first attempts by using polynomial expressions}

The stabilization of the algorithm is a task that is inherently linked with the coding of the algorithm, since it is a side effect that is not present in the analytic form of the algorithm. In order to remove the instability, we decided to try alternative ways to code the algorithm. Also, since the instability is a major problem for higher order dimensional systems, we tried to reproduce the instability even in the simple $2 \times 2$ system described above. It turned out that even in this case we had trouble when too many stages are linked together. In fact, if we consider a system with six stages and explicitly compute the determinant

$$
\operatorname{det} A_{\text {tot }}(\omega)=\prod_{i=1}^{6} A_{i}(\omega)
$$

we obtain the instability. This means that the explicit computation of this determinant is a difficult task when $\omega$ becomes too large. We then thought that the instability was due to $\omega$, and then tried to avoid using expressions containing $\omega$ explicitly until the very end, when the result, depending on $\omega$, has to be produced. We replaced each double precision variable with a polynomial expression (up to the order $q$ ) in terms of $\omega$ : for example

$$
a_{21} \rightarrow \frac{0 \omega^{0}+0 \omega^{1}+m \omega^{2}+0 \omega^{3}+\cdots+0 \omega^{q}}{1 \omega^{0}+0 \omega^{1}+\cdots+0 \omega^{q}} \rightarrow \frac{[0,0, m, 0, \ldots, 0]}{[1,0, \ldots, 0]} .
$$

That is, we split each $a_{i j}$ variable into one vector for the numerator and one vector for the denominator. We then performed the various computations in a semi-symbolic way, operating on the polynomial coefficient without involving $\omega$, until the very end. With this method we were able to explicitly compute the determinant of a six-stage system without any instability, considering only one degree of freedom, i.e. when the matrix $A$ is of order 2 . Since the explicit computation of such kind of determinant is an essential step in the computation of the TF for a system with coupled degrees of freedom (i.e. when the matrix $A$ is of order 4), we expected this stabilization method to be the correct one. Unfortunately, we also discovered that the instabilities reappear when we simulate a system with coupled degrees of freedom, and this led us to investigate other possible approaches.

\section{Algorithm stabilization: an extended precision approach}

The method we use requires the computation of very small quantities (i.e. the magnitude of the TF at high frequencies), using very big ones, since $\omega$ appears in the computation with very high powers. The obvious conclusion is that we have to keep lots of digits. We need this abnormal precision, since we do not have the explicit analytical formulae that describe our system. This is just what we want, since the numerical methods are used to get rid of these analytical formulae. However, as a drawback, we are no longer able to do any approximation 


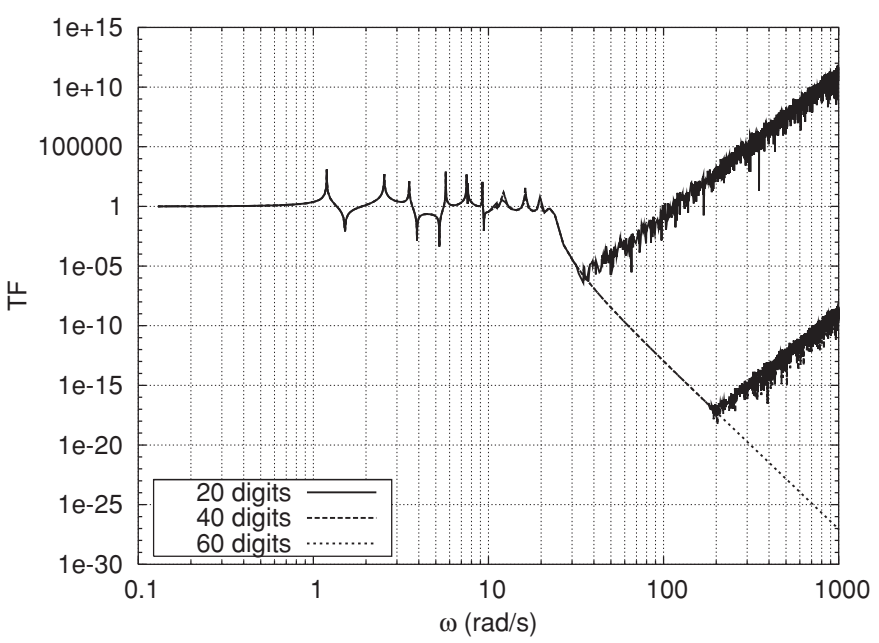

Figure 1. TF for a coupled system, i.e. with matrix $A$ of order 4 and with seven identical stages Three different curves are shown, with increasing number of digits. 60 digits precision seems to be enough to avoid any instabilities, in the frequency range of interest.

and we need to keep all the terms that appear in the process. Only at the end, when the TF is finally computed, they (almost) cancel one another out. A quantitative estimation of the number of digits required is as follows. Let $n$ be the number of digits in our code, and let $m$ be the range in the TF we have to provide as a result, that is the ratio between the highest value of the TF and the lowest one. Typically we have, for a $\mathrm{C}$ language algorithm and for our TF

$$
n=20 \quad m=20 .
$$

Then we have to take into account the fact that inside the algorithm we always have expressions of the order $\omega^{2 n_{s}}$ where $n_{s}$ is the number of stages. In order not to lose significant digits, $\omega$ should not be bigger than $\omega_{\max }$, where $\omega_{\max }$ is given by the following relation,

$$
\left(\omega_{\max }\right)^{2 n_{s}} 10^{m}=10^{n}
$$

and from this we get

$$
\omega_{\max }=1 \mathrm{rad} \mathrm{s}^{-1} \text {. }
$$

This estimation led us to the conclusion that the standard precision provided by the $\mathrm{C}$ language was not good enough for our simulation.

We then tried a 'brute force' approach ${ }^{1}$. We used a standard mathematical software (MAPLE, in our case) with a custom number of digits for our variables, a very high one indeed (of the order of 60). The results, shown in figure 1, do provide clear evidence in favour of our working hypothesis.

\section{Conclusion}

We found that an extended precision approach seems to be the key point in solving our problem. Even if the approach we used is quite simple and straightforward, there are no previous papers, to the authors' knowledge, reporting such a method. This new method, when properly verified, can model a complex suspension chain in a modular way in all six degrees of freedom. This

\footnotetext{
1 'When in doubt, use brute force.' Ken Thompson.
} 
is a topic of increasing relevance, to enhance the performance of GW detectors in the lower frequency domain. We also point out that our 'brute force' approach could even be used to enhance the stability in the polynomial approach, but there is no advantage in following this way since it is more complicated and more exposed to possible problems.

\section{Acknowledgments}

This work was supported by the Deutsche Forschungsgemeinschaft within the Sonderforschungsbereich 407. We would like to acknowledge the discussions that we had with A Viceré and G Cella during the 5th Amaldi Conference. We also thank Professor Spellucci at University of Darmstadt, and Professor Bini at University of Pisa for kind and useful discussions, in the form of long e-mails.

\section{References}

[1] Tsubono K (TAMA Collaboration) 1996 Proc. TAMA Workshop on Gravitational Wave Detection (Tokyo: Universal Academy)

[2] Bradaschia C et al 1990 Nucl. Instrum. Methods A 289518

[3] Abramovici A et al 1992 Science 256325

[4] Danzmann K et al 1994 GEO 600-Proposal for a 600m Laser-interferometric Gravitational Wave Antenna (Garching: MPQ)

[5] Saulson P R 1990 Phys. Rev. D 422437

[6] Leonhardt V, Ribichini L, Klövekorn P, Willke B, Lück H and Danzmann K 2002 Class. Quantum Grav. 191717

[7] The LIGO scientific collaboration http://xxx.lanl.gov gr-qc/0308043

[8] Viceré A 2000 Proc. Int. Summer School on Experimental Physics of Gravitational Waves (Singapore: World Scientific) p 349 